\title{
Os cem anos do átomo de Sommerfeld
}

\author{
A hundred years of Sommerfeld's atom
}

\author{
J. P. Braga* \\ Departamento de Química, Universidade Federal de Minas Gerais (31270-901) Belo Horizonte - Minas Gerais - Brasil
}

Recebido em 19 de maio, 2016. Revisado em 20 de junho, 2016. Aceito em 21 de junho, 2016

O presente trabalho discute o modelo do átomo de Sommerfeld não relativístico no contexto da mecânica quântica antiga. No intuito de deixar o trabalho completo são também discutidas as energias da partícula na caixa, rotor rígido e oscilador harmônico. Uma introdução histórica da mecânica quântica antiga e uma discussão qualitativa do correspondente modelo relativístico são também elaborados. $\mathrm{O}$ texto apresentado pode ser acompanhado por estudantes de graduação.

Palavras-chave: Átomo de Sommerfeld, Mecânica quântica ântiga, Regras de Wilson-Sommerfeld.

The present work discuss the Sommerfeld atom in the non-relativistic domain, using the old quantum mechanics formulation. The particle in a box, rigid rotor and harmonic oscillator energies are considered, to make the text complete. An historical introduction and a qualitative presentation of the relativistic formulation are also discussed. The text was prepared in a way to make it acessible to an undergraduated student.

Keywords: Sommerfeld atom, Old quantum theory, Wilson-Sommerfeld quantization rules.

\section{Introdução}

Arnold Johannes Wilhelm Sommerfeld(1869-1951) fundou uma escola respeitável de colaboradores como, Hans Bethe, Peter Debye, Werner Heisenberg e Wolfgang Pauli, todos alunos de doutorado e ganhadores do Prêmio Nobel. Na lista de outros estudantes deve-se incluir Alfred Landé (fator $g$ de Landé em espectroscopia atômica) e Wilhelm Lenz(do vetor de Laplace-Runge-Lenz). Dois outros colaboradores importantes foram Walter Heitler(estudo quântico da molécula de $\mathrm{H}_{2}$, modelo de Heitler-London) e Linus Pauling, ganhador de dois prêmios Nobel. Sommerfeld foi indicado, entre 1917 e 1950, 81 vezes ao Prêmio Nobel de Física, mais do que qualquer outro físico [1,2].

O seu curso de física em seis volumes, Mecânica Clássica, Mecânica dos Corpos Deformáveis, Eletrodinâmica, Óptica, Termodinâmica e Mecânica Estatística e Equações Diferenciais Parciais, é ampla-

\footnotetext{
*Endereço de correspondência: jpbraga@ufmg.br
}

mente usado por sua didática clara [3]. No período da mecânica quântica antiga, período entre a proposta do quantum de ação por Max Planck até o surgimento da mecânica quântica, ele escreve o texto Atombau und Spektrallinien [4, editado em 1919, e com quatro edições até 1924 . Mesmo depois do surgimento da mecânica quântiga o livro foi dividido em duas partes e chegou na sua sexta edição em 1944 [5].

A combinação dos fundamentos teóricos com aplicações práticas fez da escola de Sommerfeld única na sua época. Por exemplo, em 1909, Sommerfeld teria recusado a escrever um curso de física teórica em conjunto com Max Planck, pois achava que a física teórica deveria ser baseada também em aplicações práticas, além das teóricas. Diante dessa recusa Planck escreve a Sommerfeld: I have been thinking that we would, in a certain sense, complement each other well [2]. 
É preciso deixar claro que Sommerfeld procurava a combinação de aspectos teóricos e práticos, não evitando nenhum dos dois. O trabalho desenvolvido no átomo de Sommerfeld, como discutido no presente trabalho, deixará esse ponto evidente. $\mathrm{O}$ tratamento matemático rigoroso, do ponto de vista relativístico e não relativístco, tinha um objetivo claro: explicar as linhas adicionais no espectro atômico. Na escola de Sommerfeld o estudante era incentivado a discutir questões de economia, questões militares e aplicações tecnológicas. Uma discussão aprofundada e mais detalhada da escola de Sommerfeld pode ser encontrada na referência [2].

Entende-se por mecânica quântica antiga a teoria desenvolvida no período aproximadamente de 1900 até 1925, em que conceitos clássicos eram aplicados em conjunto com a ideia do quantum de ação [6] . Nesse mesmo período, desenvolve-se um conjunto de propostas revolucionárias que abandonavam os conceitos clássicos, como por exemplo, a lei da radiação de Rayleigh-Jeans, estudo do efeito fotoéletrico, experiência de Franck-Hertz, estudo da capacidade caloríficas a baixas temperaturas, efeito Compton, dualidade onda-partícula, princípio de exclusão de Pauli. Vários outros exemplos desse período podem ser mencionados como discutidos em livros históricos ou conceituais da teoria quântica 7,8 .

O principal objetivo do presente trabalho é o de discutir o átomo de Sommerfeld [9] no contexto da mecânica quântica antiga, com etapas que possam ser acompanhadas por estudantes de graduação. Uma discussão detalhada será apresentada no contexto não relativístico, enquanto as etapas necessárias para o entendimento do modelo relativístico serão indicadas. Os resultados obtidos pela mecânica quântica antiga para o caso da partícula na caixa, rotor rígido e oscilador harmônico são também apresentados, com o intuito de deixar o texto mais completo. A aceitação dessas energias é introduzida normalmente sem prova (exceto pela energia do átomo de Bohr) nos cursos básicos de química e física. A mecânica quântica antiga serve, portanto, para suprir essa deficiência inicial, facilitando também futuros estudos na mecânica quântica. O desenvolvimento do átomo de Bohr não será discutido, pois, além de ser um caso especial do átomo de Sommerfeld, esse desenvolvimento já foi abordado com detalhes previamente 10,11 . Entretanto, o estudo detalhado do átomo de Sommerfeld, ao contrário do estudo do átomo de Bohr, não é geralmente abordado em livros texto.

\section{Max Planck na conferência Solvay de 1911}

A importância do quantum de ação proposta por Max Planck em 1901 fica evidenciada quando, em 1911, os cientistas de maior expressão da época se encontraram na primeira Conferência Solvay, cujo tema foi The Theory of Radiation and the Quanta 12. Planck e Sommerfeld estavam presentes nessa conferência, mas há de se notar a ausência de Niels Bohr.

Planck tinha convicção de que uma explicação do quantum de ação teria de envolver a mecânica clássica. Para tentar achar um lugar da discretização na mecânica clássica, Planck apoiava-se no princípio da ação mínima, certamente o princípio mais geral da mecânica clássica com aplicações em várias áreas 13.

Quando as equações de movimento da mecânica clássica são escritas, fica implícito a validade do princípio da ação mínima. Para que a forma das equações de movimento ficasse invariante numa transformação devia-se satisfazer o teorema de que a área no espaço de fase, espaço $p \times q$, ficasse constante nesse transformação, transoformação dita canônica [14]. Planck investigou a relação teórica desse teorema com a quantização da energia, postulando que a área no espaço de fase deveria ser proporcional à constante $h$ (constante de Planck),

$$
A=\iint d p d q=h
$$

Observe que a unidade da área $[p q]=[m v v t]=\mathrm{J} \times \mathrm{s}$ $\mathrm{e}[h]=\mathrm{J} \times \mathrm{s}$ são, obviamente, as mesmas. A condição (1) quando aplicada ao oscilador harmônico, fornece $E=h \nu$, como elaborado por Planck nessa conferência. Sommerfeld estava ciente desse trabalho, mas em 1911 preocupava-se com espalhamento de raios X, como é evidenciado nos Anais da conferência. 


\section{Regras de Wilson-Sommerfeld}

Cinco anos mais tarde, certamente influenciado pelo trabalho de Planck, Sommerfeld sugere a equação,

$$
\oint p d q=n h
$$

que aparece como uma consequência natural da proposta de Planck, pois $\oint p d q$ é também a área interior à função $p=p(q)$. É muito interessante notar que essa equação foi usada por Niels Bohr no seu modelo atômico.

Atribui-se a autoria de $\oint p d q=n h$ a três cientistas, Sommerfeld, Wilson e Ishara [8, mas somente Sommerfeld aplicou a quantização da ação a problemas de espectros atômicos, fato evidente no seu livro [4]. Na verdade, as idéias vieram de Planck ao quantizar o espaço de fase ou mesmo de Niels Bohr que usou essa relação de forma intuitiva. Aqui adota-se a mesma convenção de quem vivenciou de perto a situação, Linus Pauling [6], que usa o termo, regra de Wilson-Sommerfeld.

\section{Exemplos iniciais da mecânica quântica antiga}

Para uma partícula numa caixa de tamanho $a$, temse $p$ constante, pois $E=\frac{p^{2}}{2 m}$ com energia potencial igual a zero. A aplicação da regra de WilsonSommerfeld fornece [15],

$$
\oint p d q=2 p \int_{-a / 2}^{a / 2} d x=p 2 a=n h
$$

ou $p=\frac{n h}{2 a}$. A energia total será:

$$
E=\frac{p^{2}}{2 m}=\frac{n^{2} h^{2}}{8 m a^{2}}
$$

que é o mesmo resultado quântico.

A aplicação da mecânica quântica antiga ao rotor rígido fornece,

$$
\oint p d q=\oint P_{\theta} d \theta=P_{\theta} \int_{0}^{2 \pi} d \theta=n h
$$

em que o momento angular do rotor, $P_{\theta}$, é constante. A variável $\theta$ é o ângulo associado com esse momento angular. Portanto:

$$
P_{\theta}=\frac{n_{\theta} h}{2 \pi}
$$

Para a energia obter-se-á

$$
E_{r o t}=\frac{P_{\theta}^{2}}{2 I}=\frac{n_{\theta}^{2} \hbar^{2}}{2 I}
$$

que difere do resultado quântico na interpretação do número quântico. Esse ponto será abordado novamente.

A quantização da ação será aplicada ao oscilador harmônico, cuja energia total é $E=\frac{p^{2}}{2 m}+\frac{1}{2} k x^{2}$, ou,

$$
\frac{p^{2}}{(2 m E)}+\frac{x^{2}}{\left(\frac{2 E}{k}\right)}=1
$$

Entretanto a integral a ser quantizada, $\oint p d q$, é a área da elípse, isto é,

$$
\begin{array}{r}
\quad \oint p d q=\pi \times \text { Produto dos semi-eixos } \\
=\pi\left(\frac{2 E}{k}\right)^{\frac{1}{2}}(2 m E)^{\frac{1}{2}}=2 \pi E\left(\frac{m}{k}\right)^{\frac{1}{2}}=n h
\end{array}
$$

Como a frequência clássica do oscilador harmônico vale $\nu=\frac{1}{2 \pi}\left(\frac{k}{m}\right)^{\frac{1}{2}}$ pode-se escrever, $\frac{E}{\nu}=n h$ ou

$$
E=n h \nu
$$

recuperando-se a hipótese da Planck [16].

\section{O átomo de Sommerfeld e as órbitas elípticas}

Para o cálculo das trajetórias, ou órbitas, precisase saber inicialmente o valor da dependência do ângulo de espalhamento em função da coordenada $\theta=\theta(R)$. Por $P_{\theta}=\mu R^{2} \frac{d \theta}{d t}$ e $P_{R}=\mu \frac{d R}{d t}$, juntamente com $\frac{d \theta}{d t}=\frac{d \theta}{d R} \frac{d R}{d t},[14$, retira-se,

$$
\begin{aligned}
& \frac{d \theta}{d R}=\frac{\left(\frac{d \theta}{d t}\right)}{\left(\frac{d R}{d t}\right)}=\frac{P_{\theta}}{R^{2} P_{R}} \\
& = \pm \frac{P_{\theta}}{R^{2}\left(2 \mu E+2 \mu \frac{Z e^{2}}{R}-2 \mu \frac{P_{\theta}^{2}}{R^{2}}\right)^{1 / 2}}
\end{aligned}
$$

em que a conservação da energia, $E=$ $\frac{1}{2 \mu}\left(P_{R}^{2}+\frac{P_{\theta}^{2}}{R^{2}}\right)-\frac{Z e^{2}}{R}$ foi utilizada. Substituindo, $x=\frac{1}{R},\left(d x=-\frac{1}{R^{2}} d R\right)$ e integrando-se(com o ângulo inicial igual a zero e após divisão por $\left.P_{\theta}\right)$, tem-se,

$$
\theta= \pm \int_{x_{0}}^{x} \frac{1}{\left(\frac{2 \mu E}{P_{\theta}^{2}}+\frac{2 \mu Z e^{2}}{P_{\theta}^{2}} x-x^{2}\right)^{1 / 2}} d x
$$


Essa integral tem solução analítica na forma,

$$
\begin{aligned}
& I_{a}=\int \frac{1}{\left(\alpha+\beta x+\delta x^{2}\right)^{1 / 2}} d x \\
& = \pm \frac{1}{\sqrt{-\delta}} \cos ^{-1}\left(-\frac{\beta+2 \delta x}{\sqrt{\beta^{2}-4 \alpha \delta}}\right)
\end{aligned}
$$

com, $\alpha=\frac{2 \mu E}{P_{\theta}^{2}}, \beta=\frac{2 \mu Z e^{2}}{P_{\theta}^{2}}$ e $\delta=-1$. A prova dessa integral envolve basicamente a análise do termo do qual se extrai a raiz quadrada. Após colocar $\delta$ em evidência (as constantes são absorvidas no resultado final), completa-se o quadrado e faz-se uma substituição para a obtenção da integral conhecida de arco coseno. Essa integral é desenvolvida em cursos de cálculo [17] e não será provada aqui. O resultado obtido para o ângulo será,

$$
\theta(x)= \pm \cos ^{-1}\left(-\frac{1-\frac{P_{\theta}^{2}}{\mu Z e^{2}} x}{\sqrt{\left(1+\frac{2 E P_{\theta}^{2}}{\mu Z^{2} e^{4}}\right)}}\right)
$$

Rearranjando obtém-se,

$$
R=\frac{\frac{P_{\theta}^{2}}{\mu Z e^{2}}}{1+\varepsilon \cos \theta}
$$

com,

$$
\varepsilon=\sqrt{\left(1+\frac{2 E P_{\theta}^{2}}{\mu Z^{2} e^{4}}\right)}
$$

que representa a equação de uma cônica em coordenadas polares.

Desobedecendo o contexto histórico do presente estudo, pode-se usar a mecânica quântica, $\overline{P^{2}} \theta=$ $l(l+1) \hbar^{2}$ e $E=-\frac{\mu Z^{2} e^{4}}{2 n^{2} \hbar^{2}}$ (que poderia vir do modelo de Bohr) para desenvolver,

$$
\varepsilon=\sqrt{\left(1-\frac{l(l+1)}{n^{2}}\right)}
$$

Já que $l_{\max }=n-1$, retira-se $\varepsilon=\frac{1}{n}$, que será menor do que a unidade, provando que a equação (15) representa uma elípse. Obviamente que Sommerfeld não sabia desses resultados, mas conclui que as órbitas seriam elípticas, pois estava resolvendo um problema análogo ao de Kepler, movimento de planetas ao redor do sol.
Os semi-eixos da elípse podem ser calculados por comparação com resultados elementares de geometria analítica. A equação básica para essa comparação é escrita na forma,

$$
R=\frac{\frac{b^{2}}{a}}{1+\frac{c}{a} \cos \theta}=\frac{\frac{b^{2}}{a}}{1+\sqrt{1-\frac{b^{2}}{a^{2}}} \cos \theta}
$$

Por comparação com a equação (15),

$$
\begin{aligned}
\frac{b^{2}}{a} & =\frac{P_{\Theta}^{2}}{\mu Z e^{2}} \\
\frac{b^{2}}{a^{2}} & =-\frac{2 E P_{\theta}^{2}}{\mu Z^{2} e^{4}}
\end{aligned}
$$

com solução,

$$
\begin{aligned}
a & =-\frac{Z e^{2}}{2 E} \\
b & =\frac{P_{\theta}}{\sqrt{-2 \mu E}}
\end{aligned}
$$

Observe que se $a=b$, órbitas circulares, $R=\frac{b^{2}}{a}$, recuperando-se o átomo de Bohr.

\section{Quantização radial e angular}

A aplicação da mecânica quântica antiga a átomos hidrogenóides resume-se a resolver as integrais,

$$
\begin{aligned}
I_{R} & =\oint_{C} P_{R} d R=n_{R} h \\
I_{\theta} & =\oint_{C} P_{\theta} d \theta=n_{\theta} h
\end{aligned}
$$

correspondendo aos dois graus de liberdade. Observe que $n_{R}=0,1,2 \ldots$ e $n_{\theta}=1,2,3 \ldots$. Para uso posterior define-se também o equivalente ao número quântico principal, $n=n_{R}+n_{\theta}=1,2,3 \ldots$.

Para o cálculo de $I_{R}$, com $R=R(\theta)$, desenvolvese,

$$
\begin{aligned}
& P_{R} d R=\mu \frac{d R}{d t} d R=\mu\left(\frac{d R}{d \theta} \frac{d \theta}{d t}\right)\left(\frac{d R}{d \theta} d \theta\right) \\
& =\mu\left(\frac{d R}{d \theta}\right)^{2} \frac{d \theta}{d t} d \theta=P_{\theta}\left(\frac{1}{R} \frac{d R}{d \theta}\right)^{2} d \theta
\end{aligned}
$$

em que $\frac{d \theta}{d t}=\frac{P_{\theta}}{\mu R^{2}}$ foi utilizado. Usando o resultado da trajetória, equação 15 , desenvolve-se,

$$
\begin{aligned}
& \left(\frac{1}{R} \frac{d R}{d \theta}\right)=\left(\frac{d \ln R}{d \theta}\right) \\
& =\frac{d}{d \theta}\left(\ln \left(\frac{P_{\theta}^{2}}{\mu Z e^{2}}\right)-\ln (1+\varepsilon \cos \theta)\right) \\
& =\frac{\varepsilon \operatorname{sen} \theta}{1+\varepsilon \cos \theta}
\end{aligned}
$$


Portanto,

$$
\begin{aligned}
& \oint_{C} P_{R} d R=\oint_{C} P_{\theta}\left(\frac{1}{R} \frac{d R}{d \theta}\right)^{2} d \theta \\
& =\varepsilon^{2} P_{\theta} \int_{0}^{2 \pi} \frac{\operatorname{sen}^{2} \theta}{(1+\varepsilon \cos \theta)^{2}} d \theta=P_{\theta} I_{b}
\end{aligned}
$$

com,

$$
I_{b}=\varepsilon^{2} \int_{0}^{2 \pi} \frac{\operatorname{sen}^{2} \theta}{(1+\varepsilon \cos \theta)^{2}} d \theta
$$

O desenvolvimento dessa integral deve ser feito, para que o tratamento fique completo.

\section{Uma integral intermediária}

A solução da integral $I_{b}$ será desenvolvida na forma,

$$
\begin{aligned}
I_{b} & =\varepsilon^{2} \int_{0}^{2 \pi} \frac{\operatorname{sen}^{2} \theta}{(1+\varepsilon \cos \theta)^{2}} d \theta \\
& =-\int_{0}^{2 \pi} \frac{\varepsilon^{2} \operatorname{sen}^{2} \theta}{(1+\varepsilon \cos \theta)^{2}} \frac{d(1+\varepsilon \cos \theta)}{\varepsilon \sin \theta} \\
& =-\varepsilon \int_{0}^{2 \pi} \frac{\operatorname{sen} \theta}{(1+\varepsilon \cos \theta)^{2}} d(1+\varepsilon \cos \theta)
\end{aligned}
$$

Fazendo a integração por partes com, $u=\operatorname{sen} \theta$, $v=-\frac{1}{1+\cos \theta}$, retira-se,

$$
\begin{aligned}
I_{b} & =-\varepsilon \int_{0}^{2 \pi} \frac{\operatorname{sen} \theta}{(1+\varepsilon \cos \theta)^{2}} d(1+\varepsilon \cos \theta) \\
& =-\varepsilon\left[\left.\frac{-\operatorname{sen} \theta}{1+\cos \theta}\right|_{0} ^{2 \pi}+\int_{0}^{2 \pi} \frac{\cos \theta d \theta}{1+\varepsilon \cos \theta}\right] \\
& =-\varepsilon \int_{0}^{2 \pi} \frac{\cos \theta d \theta}{1+\varepsilon \cos \theta} \\
& =\int_{0}^{2 \pi}\left(\frac{1}{1+\varepsilon \cos \theta}-1\right) d \theta \\
& =\int_{0}^{2 \pi} \frac{1}{1+\varepsilon \cos \theta} d \theta-2 \pi
\end{aligned}
$$

Uma nova integral, $I_{c}=\int_{0}^{2 \pi} \frac{1}{1+\varepsilon \cos \theta} d \theta$, deve ser trabalhada. Tem-se,

$$
I_{c}=\int_{0}^{2 \pi} \frac{1}{1+\varepsilon \cos \theta} d \theta=2 \int_{0}^{\pi} \frac{1}{1+\varepsilon \cos \theta} d \theta
$$

Usando as subsituições, $t=\tan \left(\frac{\theta}{2}\right), \cos \theta=\frac{1-t^{2}}{1+t^{2}} \mathrm{e}$ $d t=\sec ^{2}\left(\frac{\theta}{2}\right) \frac{d \theta}{2}=\left(1+t^{2}\right) \frac{d \theta}{2}$ retira-se

$$
\begin{aligned}
I_{c} & =2 \int_{0}^{\infty}\left(\frac{1+t^{2}}{1+t^{2}+\varepsilon\left(1-t^{2}\right)}\right) \frac{2 d t}{1+t^{2}} \\
& =\frac{4}{1-\varepsilon} \int_{0}^{\infty} \frac{d t}{t^{2}+\left(\sqrt{\frac{1+\varepsilon}{1-\varepsilon}}\right)^{2}} \\
& =\left.\frac{4}{1-\varepsilon} \frac{1}{\sqrt{\frac{1+\varepsilon}{1-\varepsilon}}} \tan ^{-1} \frac{t}{\sqrt{\frac{1+\varepsilon}{1-\varepsilon}}}\right|_{0} ^{\infty} \\
& =\frac{4}{1-\varepsilon} \frac{1}{\sqrt{\frac{1+\varepsilon}{1-\varepsilon}}} \frac{\pi}{2} \\
& =\frac{2 \pi}{\sqrt{1-\varepsilon^{2}}}
\end{aligned}
$$

Portanto,

$$
I_{b}=\varepsilon^{2} \int_{0}^{2 \pi} \frac{\operatorname{sen}^{2} \theta}{(1+\varepsilon \cos \theta)^{2}} d \theta=2 \pi\left(\frac{1}{\sqrt{1-\varepsilon^{2}}}-1\right)
$$

Essa integração pode ser desenvolvida de maneira mais eficiente pela teoria de resíduos, mas evitase esse tratamento para que um número maior de leitores possa acompanhar o tratamento.

\section{Parte final integração radial}

Substituindo o resultado de $I_{b}$ na equação (24),

$$
\oint_{C} P_{R} d R=2 \pi P_{\theta}\left(\frac{1}{\left(1-\varepsilon^{2}\right)^{1 / 2}}-1\right)=n_{R} h
$$

A solução da parte angular é exatamente a mesma da desenvolvida no rotor rígido ou átomo de Bohr, em que se obteve, $P_{\theta}=n_{\theta} \hbar$. Esse resultado, quando substituido na equação (31) fornece finalmente,

$$
\left(\frac{1}{\left(1-\varepsilon^{2}\right)^{1 / 2}}-1\right)=\frac{n_{R}}{n_{\theta}}
$$

\section{A energia total}

Desenvolvendo a equação 32 ,

$$
\frac{1}{\left(1-\varepsilon^{2}\right)^{1 / 2}}=1+\frac{n_{R}}{n_{\theta}}=\frac{n_{R}+n_{\theta}}{n_{\theta}}=\frac{n}{n_{\theta}}
$$

Por outro lado,

$$
\frac{1}{\left(1-\varepsilon^{2}\right)^{1 / 2}}=\frac{1}{\left(1-\frac{c^{2}}{a^{2}}\right)^{1 / 2}}=\frac{a}{\left(a^{2}-c^{2}\right)^{1 / 2}}=\frac{a}{b}
$$


mostrando que,

$$
\frac{a}{b}=\frac{n_{R}+n_{\theta}}{n_{\theta}}=\frac{n}{n_{\theta}}
$$

As órbitas serão elípticas se o número quântico radial for diferente de zero. O caso especial com $n_{R}=0$ representa novamente o átomo de Bohr, um movimento circular.

A energia total de átomos hidrogenóides na mecânica quântica antiga é nesta altura do desenvolvimento facilmente obtida pois, por (16) e $(33)$,

$$
1-\varepsilon^{2}=-\frac{2 E P_{\theta}^{2}}{\mu Z^{2} e^{4}}=\left(\frac{n_{\theta}}{n}\right)^{2}
$$

ou,

$$
E=-\frac{\mu Z^{2} e^{4}}{2 P_{\theta}^{2}}=-\frac{\mu Z^{2} e^{4}}{2\left(n_{\theta} \hbar\right)^{2}}\left(\frac{n_{\theta}}{n}\right)^{2}=-\frac{\mu Z^{2} e^{4}}{2 n^{2} \hbar^{2}}
$$

Com $n=n_{R}+n_{\theta}$,

$$
E=-\frac{\mu Z^{2} e^{4}}{2\left(n_{R}+n_{\theta}\right)^{2} \hbar^{2}}
$$

expressão obtida por Sommerfeld, resultado idêntico ao trabalho de Niels Bohr. Apesar de o resultado numérico das energias ser o mesmo, deve-se perceber que existe um número quântico adicional, $n_{R}$, em relação ao modelo de Bohr. A combinação desses dois números quânticos parecia prever o formato dos orbitais atômicos.

Já que as energias do átomo de Sommerfeld são iguais à do atomo de Bohr, quais vantagens traria o modelo de Sommerfeld para o entendimento do espectro atomico? Em termos energéticos não haveria nenhuma vantagem. Todas as previsões do àtomo de Sommerfeld seriam equivalentes à de Bohr. E isso não resolvia o problema de se entender o espectro do átomo de hidrogênio, pois sabia-se que, por exemplo, na transição de $n=2$ para $n=1$ outras linhas estavam presentes 8, 18. Nenhum dos dois modelos não relativistísco, Bohr ou Sommerfeld, poderia explicar esse fato.

\section{Discussão qualitativa dos resultados relativísticos}

Sommerfeld estava consciente da limitação do seu modelo não relativístico e na segunda parte do trabalho de 1916 desenvolve o tratamento relativístico. Seguindo o trabalho de Sommerfeld [9], enumera-se os passos principais dessa dedução:
1. A massa é substituída pela massa relativística, $m=\frac{m_{0}}{\sqrt{1-\beta^{2}}}, \operatorname{com} \beta=\frac{v}{c} ;$

2. Em analogia com a equação (11), que representa um rearranjo das equações de movimento, Sommerfeld escreve as equações de movimento relativísticas para o problema de átomos de um elétron,

$$
\frac{d^{2} \sigma}{d \theta}+\sigma=\frac{Z m_{0} e^{2}}{p^{2}} \frac{1}{\sqrt{1-\beta^{2}}}
$$

$\operatorname{com} \sigma=\frac{1}{r}$ e $p=P_{\theta}$.

3. Usa a conservação da energia total $(W), W=$ $m_{0} c^{2}\left(\frac{1}{\sqrt{1-\beta^{2}}}-1\right)-\frac{Z e^{2}}{R}$, para retirar a quantidade $\frac{1}{\sqrt{1-\beta^{2}}}$, reescrevendo a equação de movimento na forma,

$$
\begin{aligned}
& \frac{d^{2} \sigma}{d \theta}+\sigma\left(1-\left(\frac{Z e^{2}}{p c}\right)^{2}\right) \\
& =\frac{Z e^{2} m_{0}}{p^{2}}\left(1+\frac{W}{m_{0} c^{2}}\right)
\end{aligned}
$$

4. Uma solução é proposta na forma,

$$
\sigma=A \cos \gamma \theta+B \sin \gamma \theta+C
$$

Com substituição nas equações de movimento, Sommerfeld obteve $\gamma^{2}=1-\left(\frac{Z e^{2}}{p c}\right)^{2}$ e $C=$ $\frac{Z e^{2} m_{0}}{\gamma^{2} p^{2}}\left(1+\frac{W}{m_{0} c^{2}}\right)$.

5. Com rearranjo da solução obteve,

$$
\frac{1}{r}=\sigma=C(1+\varepsilon \cos \gamma \theta)
$$

em analogia com as orbitas descritas pela equação 15);

6. Usando as equações (21) e procedendo de forma análoga obteve o resultado da energia total relativística. É interessante observar que a integral envolvida nessas passagens é a mesma desenvolvida no item 7 e as passagens, apesar de trabalhosas, podem ser feitas por um estudante de graduação.

7. A energia obtida por Sommerfeld, como ora descrito, foi,

$$
E=-m_{0} c^{2}\left\{1-\left[1+\left(\frac{\alpha Z}{\left(n-n_{\theta}\right)+\left(n_{\theta}^{2}-\alpha^{2} Z^{2}\right)^{1 / 2}}\right)^{2}\right]_{(43)}^{-1 / 2}\right\}
$$

em que $m_{0}$ é a massa de repouso do eletron, $c$ a velocidade da luz e $\alpha=\frac{e^{2}}{\hbar c}$ a constante de estrutura fina. Deve-se a Sommerfeld a dedução teórica, pela primeira vez, dessa constante. 
O uso da mecânica quântica relativística fornece o resultado [19,20,

$$
E=-m_{0} c^{2}\left\{1-\left[1+\left(\frac{\alpha Z}{\left(n-\left(j+\frac{1}{2}\right)\right)+\left(\left(j+\frac{1}{2}\right)^{2}-\alpha^{2} Z^{2}\right)^{1 / 2}}\right)^{2}\right]^{-1 / 2}\right\}
$$

com $n=0,1,2 \ldots$ e $j=\frac{1}{2}, \frac{3}{2}[19]$, para a energia de átomos de um elétron. A semelhança das duas expressões é evidente, mudando apenas a interpretação dos números quânticos. As transições finas do espectro atômico, como a transição do estado $2 p_{3 / 2}$ para o estado $2 s_{1 / 2}, 2 p_{1 / 2}$ podiam ser explicadas, com a devida interpretação dos números quânticos, pela teoria relativística de Sommerfeld.

\section{Conclusões}

Os resultados da mecânica quântica antiga são surpreendentes. As energias da partículas na caixa, oscilador harmônico(exceto pela energia de ponto zero) e átomos de um életron, relativístico e não relativísticos(com a devida interpretação dos número quânticos), foram previstas corretamente pela mecânica quântica antiga. A semelhança dos resultados relativísticos com o não relativístico é também impressionante, indicando a associação $n_{\theta}=j+\frac{1}{2}$, resultado que encontra explicação no método WKB 19] e conhecido como correção de Langer 21].

De fato as regras de Wilson-Sommerfeld encontram sua explicação na teoria de perturbação na mecânica quântica. Por exemplo, a fase de espalhamento semi-clássica tem uma excelente concordância com a fase quântica [22] se a correção de Langer é levada em consideração. O mesmo efeito acontece na energia do rotor rígido, que só equivale ao resultado quântico para valores muito altos de $n_{\theta}$. Essa restrição pode ser relaxada se a associação $n_{\theta}=j+\frac{1}{2}$ é empregada. Reescrevendo a energia do rotor na forma, $E=\frac{n_{\theta}^{2}}{2 I}=\frac{\left(j+\frac{1}{2}\right)^{2}}{2 I}$, observa-se uma melhor aproximação para a energia exata, pois $\left(j+\frac{1}{2}\right)^{2}=j^{2}+j+\frac{1}{4} \approx j(j+1)$.

A energia de ponto zero era algo que faltava na energia do oscilador harmônico, mas mesmo essa energia foi também inferida na mecânica quântica antiga. Numa expansão até segunda ordem em $\frac{k T}{h \nu}$, Einstein e Stern 23] verificaram que a energia de ponto zero teria de ser considerada, num caso específico do cálculo da capacidade calorífica. Mesmo que isso não seja em geral necessário 24], a energia de ponto zero já estava sendo considerada.
Não se pode negar a simplicidade e a beleza da combinação dos resultados da mecânica clássica com o quantum de ação. No período em que a mecânica quântica antiga foi empregada, os cientistas baseavam-se na intuição para propor novas teorias. As palavras de Max Born no obituário de Sommerfeld [25], ...he developed a gift for the divining or guessing of mathematical relations from experimental data..., expressam esse sentimento e são inspiradoras. A gift for the divining or guessing ... seria a melhor maneira de entender os cientistas desse período.

\section{Agradecimento}

Gostaria de agradecer ao CNPq pela ajuda financeira

\section{Referências}

[1] M. Eckert, Arnold Sommerfeld: Science, Life and Turbulent Times 1868-1951 (Springer, New York, 2013).

[2] S. Seth, Crafting the Quantum (The MIT Press, Massachusetts, 2010).

[3] A. Sommerfeld, American Journal of Physics 17, 315 (1949).

[4] A. Sommerfeld, Atombau und Spektrallinien (F. Vieweg, Braunschweig, 1919).

[5] M. Badino and J. Navarro, A History of Quantum Physics through Its Textbooks (Max Planck Library for History and Development of Knowledge, Berlin, 2013).

[6] L. Pauling and E. Wilson, Introduction to Quantum Mechanics with Applications to Chemistry (Dover Publications, Mineola, 2012).

[7] F. Hund, The History of Quantum Theory (Harrap, London, 1974).

[8] M. Jammer, The Conceptual Development of Quantum Mechanics (McGraw-Hill, New York, 1966).

[9] A. Sommerfeld, Ann. d. Phys. 51, 1 (1916). 
[10] J.P. Braga and C.A.L. Filgueiras, Quim. Nova 36, 1073 (2013).

[11] J.P. Braga, C.A.L. Filgueiras and N.H.T. Lemes, Quim. Nova 36, 1078 (2013).

[12] J. Mehra, The Solvay Conferences on Physics: Aspects of the Development of Physics Since 1911 (Springer, The Netherlands, 2012).

[13] W. Yourgrau and S. Mandelstam, Variational Principles in Dynamics and Quantum Theory (Dover Publications, Mineola, 1979).

[14] L. Landau and E. Lifshitz, Mechanics (ButterworthHeinemann, Oxford, 1976).

[15] M. Born, The Mechanics of the Atom (G. Bell and Sons, London, 1927).

[16] J.P. Braga, Fundamentos de Química Quântica (Editora UFV, Viçosa, 2007).
[17] W. Kaplan, Cálculo Avançado (Edgard Bücher ltda., São Paulo, 1976).

[18] G. Herzberg, Atomic Spectra and Atomic Structure (Dover Publications, Mineola, 1944).

[19] A. Messiah, Quantum Mechanics (Dover Publications, Mineola, 2014).

[20] J.D. Bjorken and S.D. Drell, Relativistic Quantum Theory (McGraw Hill Company, New York, 1964).

[21] R.E. Langer, Phys. Rev. 51, 669 (1937).

[22] V.D. Viterbo, N. H. T. Lemes, and J. P. Braga, Revista Brasileira de Ensino de Física 36, 1310 (2014).

[23] A. Einstein and O. Stern, Ann. d. Phys. 40, 551 (1913).

[24] J.P. Braga, Físico-Química - Aspectos Moleculares e Fenomenológicos (Editora UFV, Viçosa, 2002).

[25] M. Born, Obituary Notices of Fellows of the Royal Society 8, 274 (1952). 